International Journal of Modern Physics A

(C) World Scientific Publishing Company

\title{
HYBRID INFLATION FOLLOWED BY MODULAR INFLATION
}

\author{
GEORGE LAZARIDES \\ Physics Division, School of Technology, Aristotle University of Thessaloniki, \\ Thessaloniki 54124, Greece \\ lazaride@eng.auth.gr
}

Received 11 June 2007

\begin{abstract}
Inflationary models with a superheavy scale F-term hybrid inflation followed by an intermediate scale modular inflation are considered. The restrictions on the power spectrum $P_{\mathcal{R}}$ of curvature perturbation and the spectral index $n_{\mathrm{s}}$ from the recent data within the power-law cosmological model with cold dark matter and a cosmological constant can be met provided that the number of e-foldings $N_{\mathrm{HI}} *$ suffered by the pivot scale $k_{*}=0.002 / \mathrm{Mpc}$ during hybrid inflation is suitably restricted. The additional e-foldings needed for solving the horizon and flatness problems are generated by modular inflation with a string axion as inflaton. For central values of $P_{\mathcal{R}}$ and $n_{\mathrm{s}}$, the grand unification scale comes out, in the case of standard hybrid inflation, close to its supersymmetric value $M_{\mathrm{GUT}} \simeq 2.86 \times 10^{16} \mathrm{GeV}$, the relevant coupling constant is relatively large $(\approx 0.005-0.14)$, and $10 \lesssim N_{\mathrm{HI} *} \lesssim 21.7$. In the shifted [smooth] hybrid inflation case, the grand unification scale can be identified with $M_{\mathrm{GUT}}$ for $N_{\mathrm{HI} *} \simeq 21\left[N_{\mathrm{HI} *} \simeq 18\right]$.
\end{abstract}

Keywords: hybrid inflation; modular inflation.

PACS number: 98.80.Cq

\section{Introduction}

Fitting the recent three-year results 1 from the Wilkinson microwave anisotropy probe satellite (WMAP3) with the standard power-law cosmological model with cold dark matter and a cosmological constant $(\Lambda \mathrm{CDM})$, one obtains 1 that, at the pivot scale $k_{*}=0.002 / \mathrm{Mpc}$,

$$
n_{\mathrm{s}}=0.958 \pm 0.016 \Rightarrow 0.926 \lesssim n_{\mathrm{s}} \lesssim 0.99
$$

at $95 \%$ confidence level. One of the most natural and well-motivated classes of inflationary models is the class 2 of supersymmetric (SUSY) F-term hybrid inflation $(\mathrm{FHI}) \sqrt{3 \sqrt{4}}$ models. They are realized at (or close to) the SUSY grand unified theory $(\mathrm{GUT})$ scale $M_{\mathrm{GUT}} \simeq 2.86 \times 10^{16} \mathrm{GeV}$. However, these inflationary models predict that the scalar spectral index $n_{\mathrm{s}}$ is too close to unity and without much running, which is in conflict with the WMAP3 data. Moreover, including supergravity (SUGRA) corrections with canonical Kähler potential, $n_{\mathrm{s}}$ gets 5 closer to unity and can even exceed it. 
One way out of this inconsistency is $6-\sqrt{8}$ to use a quasi-canonical Kähler potential with a convenient arrangement of the sign of one of its terms. This yields $7-9$ a negative mass term for the inflaton in the inflationary potential, which, thus, in general acquires a local maximum. Hilltop inflation ${ }^{6}$ can then be realized as the inflaton rolls from this maximum down to smaller values. In this case, $n_{\mathrm{s}}$ can become consistent with Eq. (11), but only at the cost of a mild tuning 9 of the initial conditions. Note, though, that, in some cases, $\frac{810}{10}$ acceptable $n_{\mathrm{s}}$ 's can be obtained even without this local maximum. Another possibility for resolving the tension between FHI and the data is $[1$ to include a small contribution to the curvature perturbation from cosmic strings, 1213 which can make $n_{\mathrm{s}}$ 's between 0.98 and 1 compatible with the data. However, the GUT scale is constrained $9|14| 15$ to values well below $M_{\mathrm{GUT}}$.

In this talk, we present a recent proposal 16 of a two-step inflationary set-up: a GUT scale FHI followed by an intermediate scale modular inflation (MI) $\frac{17}{17}$ which allows acceptable $n_{\mathrm{s}}$ 's even with canonical Kähler potential and without cosmic strings. The idea is to constrain the number of e-foldings that $k_{*}$ suffers during FHI to relatively small values, which reduces $n_{\mathrm{s}}$ to acceptable values. The additional number of e-foldings required for solving the horizon and flatness problems of standard hot big bang cosmology is naturally provided by MI, which can be easily realized by a string axion. We show that this scheme can satisfy all the relevant constraints with natural values of its parameters.

In Sec. 2, we review the basic FHI models. The calculation of their inflationary observables is described in Sec. 3. Then, in Sec. 4, we sketch the main features of MI and, in Sec. 5, we exhibit the constraints imposed on our set-up. Finally, in Sec. 6, we present our numerical results and, in Sec. 7, we summarize our conclusions.

\section{The FHI Models}

The relevant superpotentials for the various versions of FHI are 2

$$
W= \begin{cases}\kappa S\left(\bar{\Phi} \Phi-M^{2}\right) & \text { for standard FHI, } \\ \kappa S\left(\bar{\Phi} \Phi-M^{2}\right)-S \frac{(\bar{\Phi} \Phi)^{2}}{M_{\mathrm{S}}^{2}} & \text { for shifted FHI, } \\ S\left(\frac{(\bar{\Phi} \Phi)^{2}}{M_{\mathrm{S}}^{2}}-\mu_{\mathrm{S}}^{2}\right) & \text { for smooth FHI, }\end{cases}
$$

where $\bar{\Phi}, \Phi$ are left handed superfields belonging to conjugate representations of a GUT gauge group $G$ and reducing its rank by their vacuum expectation values (VEVs), $S$ is a gauge singlet left handed superfield, $M_{\mathrm{S}} \sim 5 \times 10^{17} \mathrm{GeV}$ is the string scale, and $\kappa$ and $M, \mu_{\mathrm{S}}\left(\sim M_{\mathrm{GUT}}\right)$ are made real and positive by field redefinitions.

The superpotential for standard $\frac{3 \sqrt{4}}{\mathrm{FHI}}$ in Eq. (2) is the most general renormalizable superpotential consistent with a global U(1) R symmetry 4 under which

$$
S \rightarrow e^{i \alpha} S, \quad \bar{\Phi} \Phi \rightarrow \bar{\Phi} \Phi
$$

Note, in passing, that global continuous symmetries such as this R symmetry can effectively arise $\frac{18}{18}$ from the rich discrete symmetry groups encountered in many 
compactified string theories (see e.g. Ref. 19). Including in the superpotential for standard FHI the leading non-renormalizable term, one obtains the superpotential for shifted ${ }^{20} \mathrm{FHI}$ in Eq. (2). The superpotential for smooth ${ }^{21} \mathrm{FHI}$ is produced by further imposing a $Z_{2}$ symmetry under which $\Phi \rightarrow-\Phi$ and, thus, allowing only even powers of $\bar{\Phi} \Phi$.

The vanishing of the D-terms implies that $|\langle\bar{\Phi}\rangle|=|\langle\Phi\rangle|$, while the vanishing of the F-terms gives the VEVs of the fields in the SUSY vacuum, namely $\langle S\rangle=0$ and $|\langle\bar{\Phi}\rangle|=|\langle\Phi\rangle| \equiv v_{G}$ with

$$
v_{G}= \begin{cases}M & \text { for standard FHI, } \\ \frac{M}{\sqrt{2 \xi}} \sqrt{1-\sqrt{1-4 \xi}} & \text { for shifted FHI, } \\ \sqrt{\mu_{\mathrm{S}} M_{\mathrm{S}}} & \text { for smooth FHI, }\end{cases}
$$

where $\xi \equiv M^{2} / \kappa M_{\mathrm{S}}^{2}$ with $1 / 7.2<\xi<1 / 4.20$ So, the $W$ 's in Eq. (2) lead to the spontaneous breaking of $G$. The same superpotentials give rise to hybrid inflation. This is due to the fact that, for large enough values of $|S|$, there exist flat directions in field space, i.e. valleys of local minima of the classical potential with constant (or almost constant in the case of smooth FHI) potential energy density, which can serve as inflationary paths.

The dominant contribution to the (inflationary) potential energy density along these paths is

$$
V_{\mathrm{HI} 0}= \begin{cases}\kappa^{2} M^{4} & \text { for standard FHI, } \\ \kappa^{2} M_{\xi}^{4} & \text { for shifted FHI, } \\ \mu_{\mathrm{S}}^{4} & \text { for smooth FHI, }\end{cases}
$$

where $M_{\xi} \equiv M \sqrt{1 / 4 \xi-1}$. For inflation to be realized, we need a slope along the flat direction (inflationary valley) to drive the inflaton towards the vacuum. In the cases of standard $\frac{4}{4}$ and shifted ${ }^{20}$ FHI, this slope is generated by the SUSY breaking on this valley caused by the non-vanishing $V_{\mathrm{HI}}$ on the valley. This gives rise to logarithmic radiative corrections to the potential. On the other hand, in the case of smooth $\frac{21}{\mathrm{FHI}}$, the inflationary valley is not classically flat and, thus, there is no need of radiative corrections. The relevant correction $V_{\text {HIc }}$ to the inflationary potential can be written as follows:

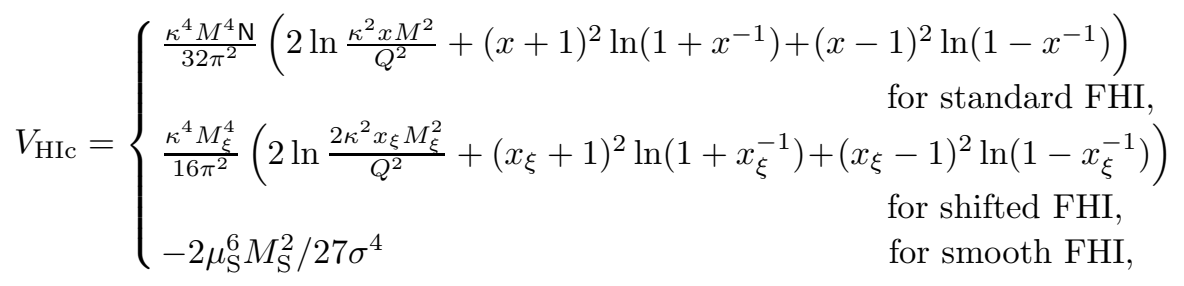

where $\sigma \equiv \sqrt{2}|S|$ is the canonically normalized inflaton field, $\mathrm{N}$ is the dimensionality of the representations to which $\bar{\Phi}$ and $\Phi$ belong in the case of standard FHI, $Q$ is a renormalization scale, $x \equiv|S|^{2} / M^{2}$, and $x_{\xi} \equiv \sigma^{2} / M_{\xi}^{2}$. For minimal Kähler 
potential, the leading SUGRA correction to the inflationary potential reads 31515

$$
V_{\mathrm{HIS}}=V_{\mathrm{HI0}} \frac{\sigma^{4}}{8 m_{\mathrm{P}}^{4}},
$$

where $m_{\mathrm{P}} \simeq 2.44 \times 10^{18} \mathrm{GeV}$ is the reduced Planck scale. In the case of standard FHI, the contribution 22 to the inflationary potential from the soft SUSY breaking terms is negligibly small in our set-up due to the large $\kappa$ 's encountered (see Sec. 6). This contribution, in general, does not have 22 a significant effect in the cases of shifted and smooth FHI too. All in all, the general form of the potential which drives the various versions of FHI reads

$$
V_{\mathrm{HI}} \simeq V_{\mathrm{HIO}}+V_{\mathrm{HIc}}+V_{\mathrm{HIS}}
$$

During standard FHI, both $\bar{\Phi}$ and $\Phi$ vanish and so the GUT gauge group $G$ is restored. As a consequence, topological defects such as cosmic strings, 1213 magnetic monopoles $\frac{23 \mid 24}{24}$ or domain walls $\sqrt{1325}$ will be copiously produced $\frac{21}{21}$ via the Kibble

mechanism 26 during the spontaneous breaking of $G$ at the end of FHI if they are predicted by this symmetry breaking. This, which could lead to a cosmological catastrophe in the cases of monopoles and walls, is avoided in shifted and smooth FHI, since the form of $W$ allows the existence of non-trivial inflationary valleys along which $G$ is spontaneously broken (with $\bar{\Phi}$ and $\Phi$ acquiring non-zero values). Therefore, no topological defects are produced in these cases. In standard FHI, on the other hand, we must normally ensure that no monopoles or walls are predicted by the underlying particle physics scheme. In our set-up, however, this restriction can be evaded since the subsequent MI dilutes the topological defects.

\section{The Dynamics of FHI}

We will assume that all the cosmological scales cross outside the horizon during FHI and do not re-enter the horizon before the onset of MI (see below). The latter guarantees that they are not "re-processed" by MI. So, we can apply the standard formalism (see e.g. Ref. 27) to calculate the inflationary observables of FHI. Namely, the number of e-foldings $N_{\mathrm{HI} *}$ that the pivot scale $k_{*}$ suffers during FHI is given by

$$
N_{\mathrm{HI} *}=\frac{1}{m_{\mathrm{P}}^{2}} \int_{\sigma_{\mathrm{f}}}^{\sigma_{*}} d \sigma \frac{V_{\mathrm{HI}}}{V_{\mathrm{HI}}^{\prime}},
$$

where the prime denotes derivation with respect to $\sigma, \sigma_{*}$ is the value of $\sigma$ when $k_{*}$ crosses outside the horizon of FHI, and $\sigma_{\mathrm{f}}$ is the value of $\sigma$ at the end of FHI. In the slow-roll approximation, $\sigma_{\mathrm{f}}$ is found from the condition

$$
\begin{gathered}
\max \left\{\epsilon\left(\sigma_{\mathrm{f}}\right),\left|\eta\left(\sigma_{\mathrm{f}}\right)\right|\right\}=1, \quad \text { where } \\
\epsilon \simeq \frac{m_{\mathrm{P}}^{2}}{2}\left(\frac{V_{\mathrm{HI}}^{\prime}}{V_{\mathrm{HI}}}\right)^{2} \text { and } \eta \simeq m_{\mathrm{P}}^{2} \frac{V_{\mathrm{HI}}^{\prime \prime}}{V_{\mathrm{HI}}}
\end{gathered}
$$

In standard $\sqrt{4}$ and shifted 20 FHI, the end of inflation coincides with the onset of the GUT phase transition, i.e. the slow-roll conditions are violated infinitesimally close 
to the critical point at $\sigma=\sigma_{\mathrm{c}} \equiv \sqrt{2} M\left[\sigma=\sigma_{\mathrm{c}} \equiv M_{\xi}\right]$ for standard [shifted] FHI, where the inflationary path is destabilized and the "waterfall" regime commences. On the contrary, the end of smooth ${ }^{21} \mathrm{FHI}$ is not abrupt since the inflationary path is stable with respect to variations in $\bar{\Phi}, \Phi$ for all $\sigma$ 's and $\sigma_{\mathrm{f}}$ is found from Eq. (10).

The power spectrum $P_{\mathcal{R}}$ of the curvature perturbation at $k_{*}$ is given by

$$
P_{\mathcal{R}}^{1 / 2}=\left.\frac{1}{2 \sqrt{3} \pi m_{\mathrm{P}}^{3}} \frac{V_{\mathrm{HI}}^{3 / 2}}{\left|V_{\mathrm{HI}}^{\prime}\right|}\right|_{\sigma=\sigma_{*}} .
$$

Finally, the spectral index $n_{\mathrm{s}}$ and its running $d n_{\mathrm{S}} / d \ln k$ are

$$
\begin{aligned}
n_{\mathrm{s}} & =1-6 \epsilon\left(\sigma_{*}\right)+2 \eta\left(\sigma_{*}\right) \quad \text { and } \\
d n_{\mathrm{s}} / d \ln k & =2\left(4 \eta\left(\sigma_{*}\right)^{2}-\left(n_{\mathrm{s}}-1\right)^{2}\right) / 3-2 \xi\left(\sigma_{*}\right)
\end{aligned}
$$

respectively with $\xi \simeq m_{\mathrm{P}}^{4} V_{\mathrm{HI}}^{\prime} V_{\mathrm{HI}}^{\prime \prime \prime} / V_{\mathrm{HI}}^{2}$.

\section{The Basics of MI}

After the gravity mediated soft SUSY breaking, the potential for MI is 17

$$
V_{\mathrm{MI}}=V_{\mathrm{MI} 0}-\frac{1}{2} m_{s}^{2} s^{2}+\ldots,
$$

where $s$ is the canonically normalized real string axion field, the ellipsis denotes terms which stabilize $V_{\mathrm{MI}}$ at $s \sim m_{\mathrm{P}}$,

$$
V_{\mathrm{MI} 0}=v_{s}\left(m_{3 / 2} m_{\mathrm{P}}\right)^{2}, \quad \text { and } m_{s} \sim m_{3 / 2}
$$

with $m_{3 / 2} \sim 1 \mathrm{TeV}$ being the gravitino mass and the dimensionless parameter $v_{s}$ being of order unity, which yields $V_{\mathrm{MI0}}^{1 / 4} \simeq 3 \times 10^{10} \mathrm{GeV}$. In this model, inflation can be of the fast-roll type. 28 The field evolution is given 28 by

$$
s \simeq s_{\mathrm{i}} e^{F_{s} \Delta N_{\mathrm{MI}}} \quad \text { with } \quad F_{s} \equiv \sqrt{\frac{9}{4}+\left(\frac{m_{s}}{H_{s}}\right)^{2}}-\frac{3}{2},
$$

where $s_{\mathrm{i}}$ is the initial value of $s$ (at the onset of MI), $H_{s} \simeq \sqrt{V_{\mathrm{MIO}}} / \sqrt{3} m_{\mathrm{P}}$ is the Hubble parameter corresponding to $V_{\mathrm{MI}}$, and $\Delta N_{\mathrm{MI}}$ is the number of e-foldings obtained from $s=s_{\mathrm{i}}$ until a given $s$.

From Eq. (15), we estimate the number of e-foldings $N_{\mathrm{MI}}$ during MI:

$$
N_{\mathrm{MI}} \simeq \frac{1}{F_{s}} \ln \left(\frac{s_{\mathrm{f}}}{s_{\mathrm{i}}}\right),
$$

where $s_{\mathrm{f}}=\min \left\{\langle s\rangle, s_{\mathrm{sr}}\right\}$ is the final value of $s$ with $\langle s\rangle \sim m_{\mathrm{P}}$ being the VEV of $s$ and $s_{\mathrm{sr}}$ determined by the condition

$$
\epsilon_{\mathrm{MI}} \equiv-\frac{\dot{H}_{\mathrm{MI}}}{H_{\mathrm{MI}}^{2}} \simeq \frac{1}{2} F_{s}^{2}\left(\frac{s}{m_{\mathrm{P}}}\right)^{2}=1
$$

( $H_{\mathrm{MI}}$ is the Hubble parameter during $\mathrm{MI}$ and the dot denotes derivation with respect to the cosmic time). For definiteness, we take $\langle s\rangle=m_{\mathrm{P}}$ throughout our calculation. 


\section{Observational Constraints}

Our scenario needs to satisfy the following constraints:

(i) The power spectrum in Eq. (11) is to be confronted with the WMAP3 data 1 .

$$
P_{\mathcal{R}}^{1 / 2} \simeq 4.86 \times 10^{-5} \text { at } \quad k_{*}=0.002 / \mathrm{Mpc}
$$

(ii) In our case, the horizon and flatness problems of big bang cosmology can be resolved provided that the total number of e-foldings $N_{\text {tot }}$ suffered by $k_{*}$ is given 329 by

$$
N_{\text {tot }} \simeq 22.6+\frac{2}{3} \ln \frac{V_{\mathrm{HIO}}^{1 / 4}}{1 \mathrm{GeV}}+\frac{1}{3} \ln \frac{T_{\mathrm{Mrh}}}{1 \mathrm{GeV}},
$$

where $T_{\mathrm{Mrh}}$ is the reheat temperature after the completion of MI. Here, we have assumed that the reheat temperature after FHI is lower than $V_{\text {MI0 }}^{1 / 4}$ and, thus, the whole inter-inflationary period is matter dominated. In our set-up, $N_{\text {tot }}$ consists of two contributions:

$$
N_{\text {tot }}=N_{\mathrm{HI} *}+N_{\mathrm{MI}}
$$

(iii) The assumption that all the cosmological scales leave the horizon during FHI and do not re-enter the horizon before the onset of MI yields 2930 the restriction:

$$
N_{\mathrm{HI} *} \gtrsim N_{\mathrm{HI} *}^{\min } \simeq 3.9+\frac{1}{6} \ln \frac{V_{\mathrm{HI} 0}}{V_{\mathrm{MI} 0}} .
$$

The first term in the expression for $N_{\mathrm{HI} *}^{\min }$ is the number of e-foldings elapsed between the horizon crossing of the pivot scale $k_{*}$ and the scale $0.1 / \mathrm{Mpc}$ during FHI. Length scales $\sim 10 \mathrm{Mpc}$ are starting to feel non-linear effects and it is, thus, difficult to constrain 30 primordial density fluctuations on smaller length scales. So, we take the largest cosmological scale to be about $0.1 / \mathrm{Mpc}$.

(iv) In the FHI models, $\left|d n_{\mathrm{s}} / d \ln k\right|$ increases 31 as $N_{\mathrm{HI} *}$ decreases. Therefore, consistency with the assumptions of the power-law $\Lambda$ CDM cosmological model, which requires that

$$
\left|d n_{\mathrm{s}} / d \ln k\right| \ll 0.01,
$$

yields a lower bound on $N_{\mathrm{HI} *}$. In our numerical investigation (see Sec. 6), we display boundary curves for $d n_{\mathrm{s}} / d \ln k=-0.005$ and -0.01 .

(v) The requirement of naturalness of MI constrains the dimensionless parameter $v_{s}$ in Eq. (14) as follows:

$$
0.5 \leq v_{s} \leq 10 \Rightarrow 2.45 \gtrsim m_{s} / H_{s} \gtrsim 0.55
$$

where we take $m_{s}=m_{3 / 2}$ (see below). The lower bound on $v_{s}$ guarantees that the sum of the two explicitly displayed terms in the right hand side of Eq. (13) is positive for $s<m_{\mathrm{P}}$. From Eq. (17), we see that, for the values of $m_{s} / H_{s}$ in Eq. (23), $s_{\mathrm{sr}}>m_{\mathrm{P}}$ and, thus, $s_{\mathrm{f}}=m_{\mathrm{P}}$. Eqs. (15)-(17) are not very accurate near the upper bound on $m_{s} / H_{s}$ since, in this region, the value of $\epsilon_{\mathrm{MI}}$ 
at $s=m_{\mathrm{P}}$ gets too close to unity and, thus, the Hubble parameter does not remain constant as $s$ approaches $m_{\mathrm{P}}$. So, our results at large values of $m_{s} / H_{s}$ should be considered only as indicative. Fortunately, the interesting solutions with $n_{\mathrm{s}}$ near its central value and, in the smooth FHI case, $v_{G} \sim M_{\mathrm{GUT}}$ lie near the lower bound on $m_{s} / H_{s}$, where the accuracy of these formulas is much better. Moreover,

$$
\eta_{\mathrm{MI}} \equiv m_{\mathrm{P}}^{2} \frac{V_{\mathrm{MI}}^{(2)}}{V_{\mathrm{MI}}} \simeq-\frac{1}{3}\left(\frac{m_{s}}{H_{s}}\right)^{2} \lesssim 1 \quad \text { for } \quad m_{s} / H_{s} \lesssim 1.73,
$$

where we again take $m_{s}=m_{3 / 2}$ and the superscript $(n)$ denotes the $n$-th derivative with respect to $s$. So, the interesting solutions correspond to slowrather than fast-roll MI. The unspecified terms in the ellipsis in the right hand side of Eq. (13) also generate an uncertainty in Eqs. (15)-(17), which will be assumed negligible.

(vi) Finally, we assume that FHI lasts long enough so that the almost massless string axion field $s$ is completely randomized 32 by its quantum fluctuations from FHI. We further assume that

$$
V_{\mathrm{MI} 0} \lesssim H_{\mathrm{HI} 0}^{4}
$$

where $H_{\mathrm{HIO}}=\sqrt{V_{\mathrm{HIO}}} / \sqrt{3} m_{\mathrm{P}}$ is the Hubble parameter corresponding to $V_{\mathrm{HIO}}$, so that all the values of $s$ belong to the randomization region. 32 The field $s$ remains practically frozen during the inter-inflationary period since the Hubble parameter is larger than its mass. So, all the initial values $s_{\mathrm{i}}$ of $s$ from zero to $m_{\mathrm{P}}$ are equally probable. However, we take $s_{\mathrm{i}} \gg H_{\mathrm{HI}} / 2 \pi$ so that the homogeneity of our present universe is not jeopardized by the quantum fluctuations of $s$ from FHI. Randomization of the value of a scalar field via inflationary quantum fluctuations requires that this field remains almost massless during inflation. For this, it is important that the field does not acquire ${ }^{3 \mid 33}$ mass of the order of the Hubble parameter via the SUGRA scalar potential. This is, indeed, the case for the string axion during FHI (and the inter-inflationary period).

\section{Numerical Results}

For standard 4 FHI, we take $\mathrm{N}=2$, which corresponds to the left-right symmetric GUT gauge group $\mathrm{SU}(3)_{\mathrm{c}} \times \mathrm{SU}(2)_{\mathrm{L}} \times \mathrm{SU}(2)_{\mathrm{R}} \times \mathrm{U}(1)_{\mathrm{B}-\mathrm{L}}$ with $\bar{\Phi}$ and $\Phi$ being $\mathrm{SU}(2)_{\mathrm{R}}$ doublets with $B-L=-1$ and 1 respectively. No cosmic strings are produced 34 during this realization of standard FHI, which liberates the model from extra restrictions on its parameters (for such restrictions, see e.g. Refs. 14, 15). For shifted ${ }^{20}$ FHI, the GUT gauge group is the Pati-Salam group 35 $\mathrm{SU}(4)_{\mathrm{c}} \times \mathrm{SU}(2)_{\mathrm{L}} \times \mathrm{SU}(2)_{\mathrm{R}}$. This predicts the existence of doubly charged 36 magnetic monopoles which are, though, not produced at the end of inflation as mentioned in Sec. 2. We take $T_{\mathrm{Mrh}}=1 \mathrm{GeV}$ and $m_{3 / 2}=m_{s}=1 \mathrm{TeV}$ throughout. These are indicative values, which do not affect crucially our results. Finally, we 

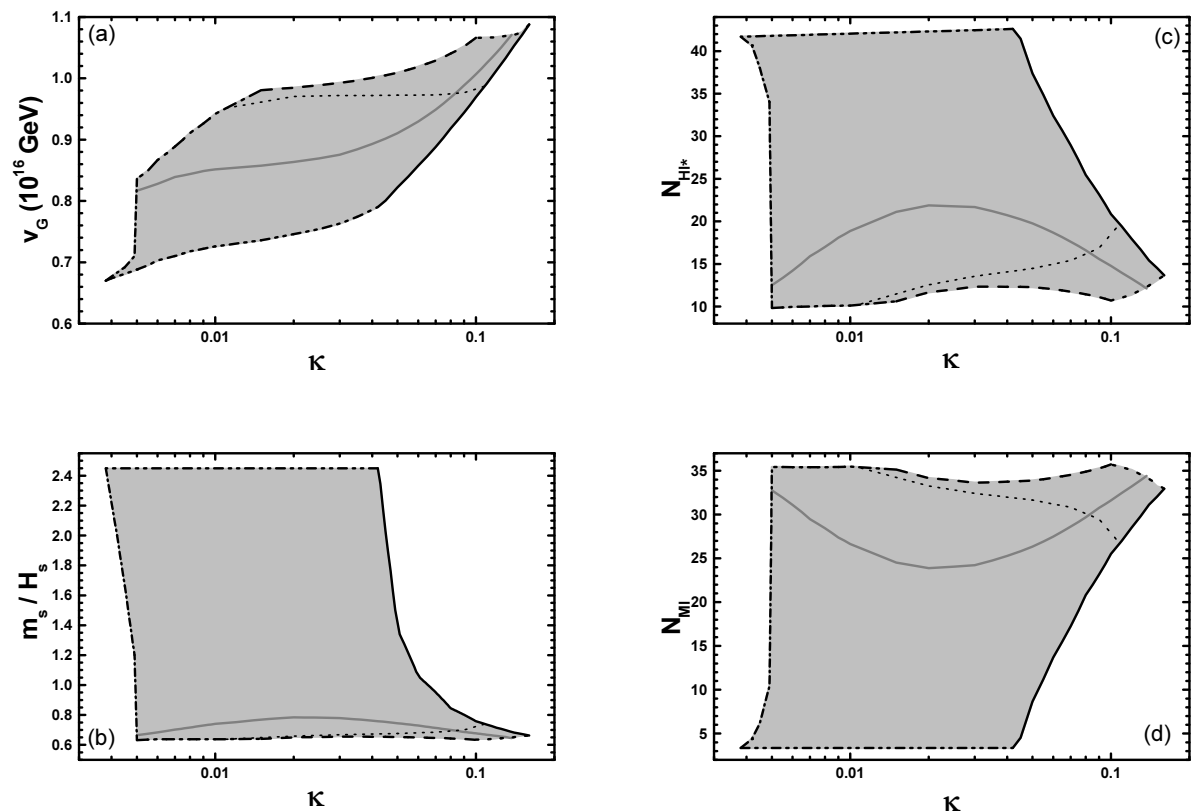

Fig. 1. Allowed (lightly gray shaded) regions in the (a) $\kappa-v_{G}$, (b) $\kappa-m_{s} / H_{s}$, (c) $\kappa-N_{\mathrm{HI} *}$, and (d) $\kappa-N_{\mathrm{MI}}$ plane for standard FHI. The black solid [dashed] lines correspond to the upper [lower] bound on $n_{\mathrm{s}}$ in Eq. (1), whereas the gray solid lines correspond to its central value in this equation. The dot-dashed [double dot-dashed] lines correspond to the lower [upper] bound on $N_{\mathrm{HI} *}\left[m_{s} / H_{s}\right.$ ] from Eq. (21] [Eq. [23)]. The bold [faint] dotted lines correspond to $d n_{\mathrm{s}} / d \ln k=$ $-0.01\left[d n_{\mathrm{s}} / d \ln k=-0.005\right]$. Finally, the lower bound on $V_{\mathrm{HIO}}$ from Eq. (25) is represented by the short dash-dotted lines.

choose the initial value $s_{\mathrm{i}}$ of the string axion $s$ at the onset of MI to be given by $s_{\mathrm{i}}=0.01 m_{\mathrm{P}}$ in all the cases that we consider. This value is close enough to $m_{\mathrm{P}}$ to have a non-negligible probability to be achieved by the randomization of $s$ during FHI. At the same time, it is adequately smaller than $m_{\mathrm{P}}$ to guarantee good accuracy of Eqs. (15)-(17) near the interesting solutions and justify the fact that we neglect the uncertainty from the ellipsis in Eq. (13). Moreover, larger $s_{\mathrm{i}}$ 's lead to smaller parameter space for interesting solutions (with $n_{\mathrm{s}}$ near its central value).

Our input parameters are $\kappa$ (for standard and shifted FHI with fixed $M_{\mathrm{S}}=$ $5 \times 10^{17} \mathrm{GeV}$ ) or $M_{\mathrm{S}}$ (for smooth FHI) and $\sigma_{*}$. Using Eqs. (12) and (18), we extract $n_{\mathrm{S}}$ and $v_{G}$ respectively. For every chosen $\kappa$ or $M_{\mathrm{S}}$, we then restrict $\sigma_{*}$ so as to achieve $n_{\mathrm{s}}$ in the range of Eq. (1) and take the output values of $N_{\mathrm{HI} *}$. Finally, we find, from Eqs. (19) and (20), the required $N_{\mathrm{MI}}$ and the corresponding $v_{s}$ or $m_{s} / H_{s}$ from Eq. (16).

Our numerical results for the three versions of FHI are presented in Figs. 13 , In Fig. 2(a) [Fig. 3(a)], we focus on a limited range of $\kappa$ 's $\left[M_{\mathrm{S}}\right.$ 's] for the sake of clarity of the presentation. Let us discuss each case separately: 

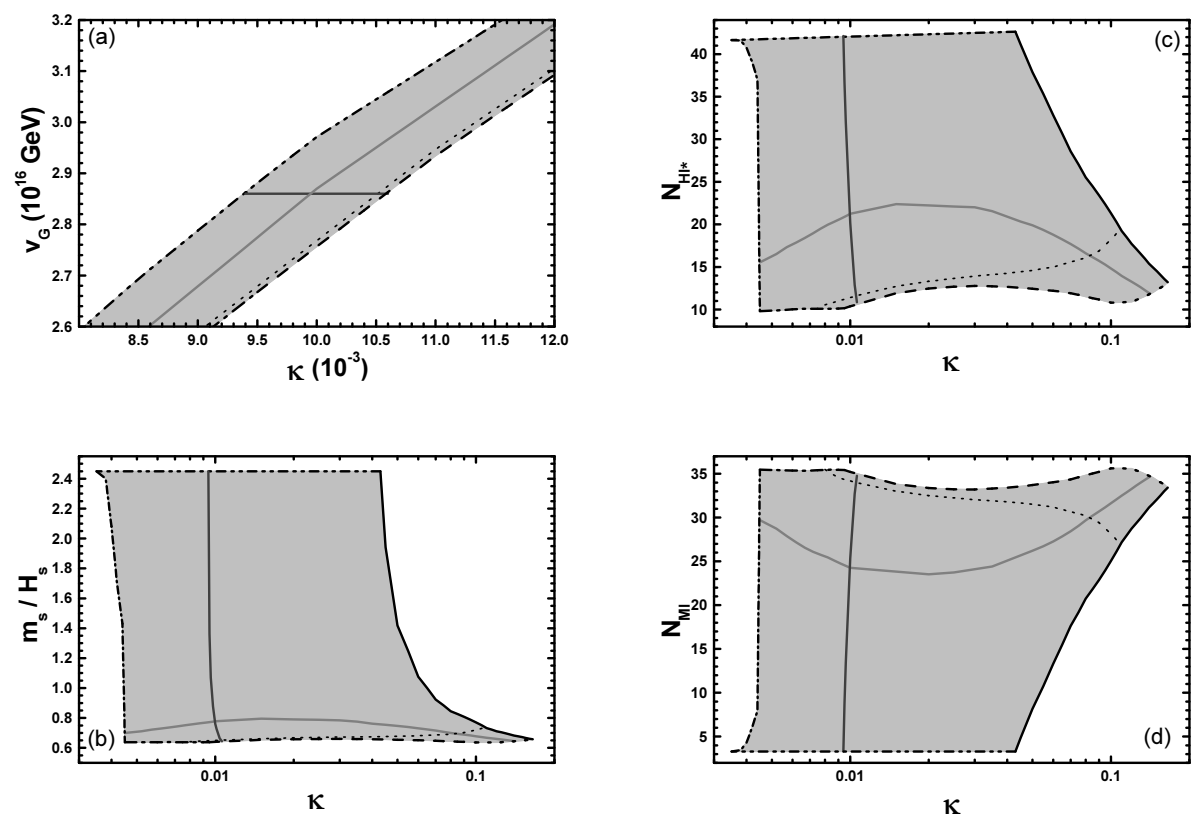

Fig. 2. Allowed regions in the (a) $\kappa-v_{G}$, (b) $\kappa-m_{s} / H_{s}$, (c) $\kappa-N_{\mathrm{HI} *}$, and (d) $\kappa-N_{\mathrm{MI}}$ plane for shifted FHI with $M_{\mathrm{S}}=5 \times 10^{17} \mathrm{GeV}$. Same notation as in Fig. 1. We also include dark gray solid lines corresponding to $v_{G}=M_{\mathrm{GUT}}$.

\subsection{Standard FHI}

In Fig. 1, we present the regions allowed by Eqs. (10), (18)-(23), and (25) in the (a) $\kappa-v_{G}$, (b) $\kappa-m_{s} / H_{s}$, (c) $\kappa-N_{\mathrm{HI} *}$, and (d) $\kappa-N_{\mathrm{MI}}$ plane for standard FHI. We observe the following:

(i) The resulting $v_{G}$ 's and $\kappa$ 's are restricted to rather large values compared to those allowed within the conventional set-up, i.e. the pure standard FHI without the complementary MI (compare with Refs. 15, 22).

(ii) As $\kappa$ increases above 0.01, the SUGRA corrections become more and more significant.

(iii) As $\kappa$ decreases below about 0.015 [0.042], the constraint from the lower [upper] bound on $n_{\mathrm{s}}$ ceases to restrict the parameters, since it is overshadowed by the lower [upper] bound on $N_{\mathrm{HI} *}\left[m_{s} / H_{s}\right]$ in Eq. (21) [Eq. (23)].

(iv) The running $d n_{\mathrm{s}} / d \ln k$ of the spectral index satisfies comfortably the bound in Eq. (22) in the largest part of the regions allowed by the other constraints, whereas $-0.005 \gtrsim d n_{\mathrm{s}} / d \ln k \gtrsim-0.01$ in a very limited part of these regions.

(v) For $n_{\mathrm{S}}=0.958$, we obtain $0.004 \lesssim \kappa \lesssim 0.14,0.79 \lesssim v_{\mathrm{G}} /\left(10^{16} \mathrm{GeV}\right) \lesssim 1.08$, and $-0.002 \gtrsim d n_{\mathrm{s}} / d \ln k \gtrsim-0.01$. Also, $10 \lesssim N_{\mathrm{HI} *} \lesssim 21.7,35 \gtrsim N_{\mathrm{MI}} \gtrsim 24$, and $0.64 \lesssim m_{s} / H_{s} \lesssim 0.77$. 

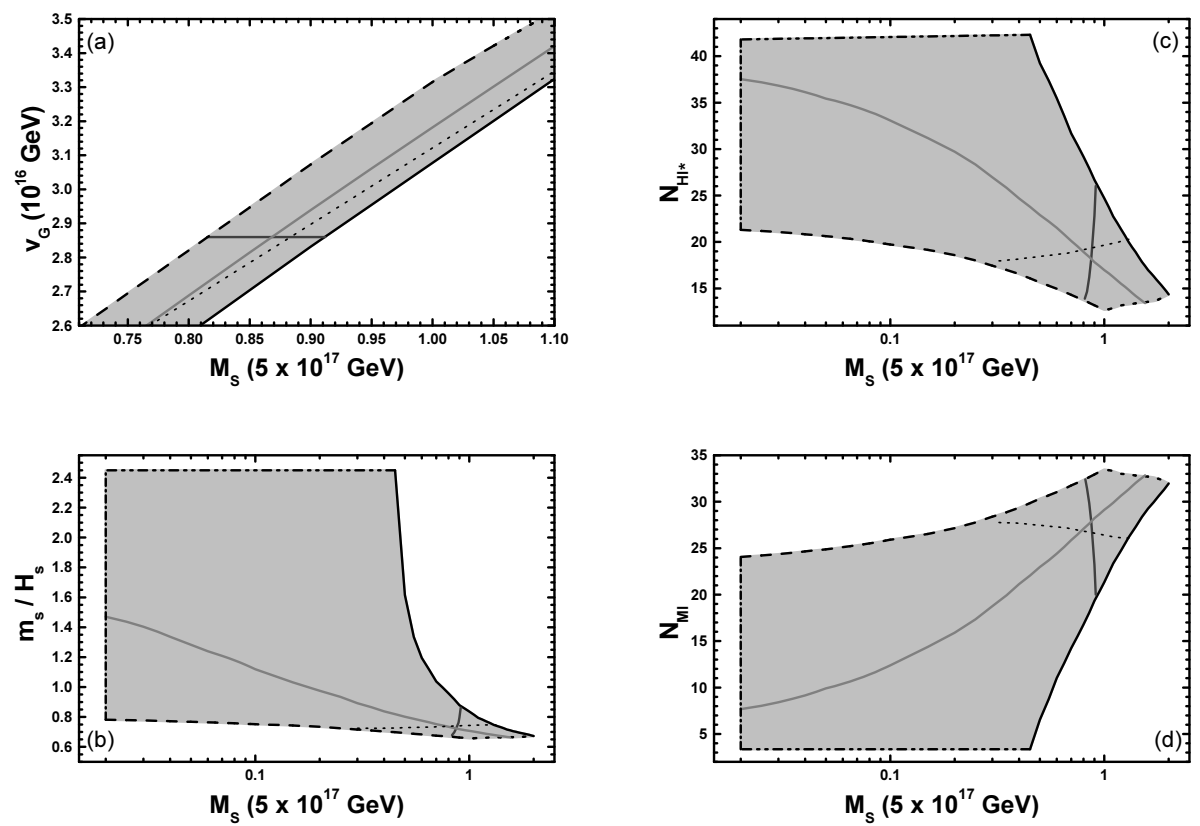

Fig. 3. Allowed regions in the (a) $M_{\mathrm{S}}-v_{G}$, (b) $M_{\mathrm{S}}-m_{s} / H_{s}$, (c) $M_{\mathrm{S}}-N_{\mathrm{HI} *}$, and (d) $M_{\mathrm{S}}-N_{\mathrm{MI}}$ plane for smooth FHI. Same notation as in Fig. 2 We included small $M_{\mathrm{S}}$ 's of less physical interest just to show the effect of the constraints.

\subsection{Shifted FHI}

In Fig. 2, we delineate the regions allowed by Eqs. (1), (18)-(23), and (25) in the (a) $\kappa-v_{G}$, (b) $\kappa-m_{s} / H_{s}$, (c) $\kappa-N_{\mathrm{HI} *}$, and (d) $\kappa-N_{\mathrm{MI}}$ plane for shifted FHI with $M_{\mathrm{S}}=5 \times 10^{17} \mathrm{GeV}$. Some observations are in order:

(i) The lower [upper] bound on $N_{\mathrm{HI} *}\left[m_{s} / H_{s}\right]$ in Eq. (21) [Eq. (23)] gives a lower [upper] bound on $v_{G}$ for each $\kappa$, in contrast to the case of standard FHI.

(ii) The results on $m_{s} / H_{s}, N_{\mathrm{HI} *}$, and $N_{\mathrm{MI}}$ are quite similar to those obtained in the case of standard FHI.

(iii) The common magnitude $v_{G}$ of the VEVs of $\bar{\Phi}$ and $\Phi$ comes out considerably larger than in the case of standard FHI and can be put equal to the SUSY GUT scale. Some key inputs and outputs for the interesting case with $v_{G}=M_{\mathrm{GUT}}$ and $n_{\mathrm{s}}=0.958$ are presented in Table 1 .

\subsection{Smooth FHI}

In Fig. 3, we present the regions allowed by Eqs. (11), (18)-(23), and (25) in the (a) $M_{\mathrm{S}}-v_{G}$, (b) $M_{\mathrm{S}}-m_{s} / H_{s}$, (c) $M_{\mathrm{S}}-N_{\mathrm{HI} *}$, and (d) $M_{\mathrm{S}}-N_{\mathrm{MI}}$ plane for smooth FHI. We observe the following: 
Table 1. Input and output parameters for our scenario with shifted $\left(M_{\mathrm{S}}=5 \times 10^{17} \mathrm{GeV}\right)$ or smooth FHI for $n_{\mathrm{s}}=0.958$ and $v_{G}=M_{\mathrm{GUT}}$.

\begin{tabular}{lrlr}
\hline \multicolumn{2}{c}{ Shifted FHI } & \multicolumn{2}{c}{ Smooth FHI } \\
\hline$\sigma_{*}\left(10^{16} \mathrm{GeV}\right)$ & 2.2 & $\sigma_{*}\left(10^{16} \mathrm{GeV}\right)$ & 23.53 \\
$\kappa$ & 0.01 & $M_{\mathrm{S}}\left(5 \times 10^{17} \mathrm{GeV}\right)$ & 0.87 \\
\hline$M\left(10^{16} \mathrm{GeV}\right)$ & 2.35 & $\mu_{\mathrm{S}}\left(10^{16} \mathrm{GeV}\right)$ & 0.188 \\
$1 / \xi$ & 4.54 & $\sigma_{\mathrm{f}}\left(10^{16} \mathrm{GeV}\right)$ & 13.42 \\
$N_{\mathrm{HI} *}$ & 21 & $N_{\mathrm{HI} *}$ & 18 \\
$d n_{\mathrm{s}} / d \ln k$ & -0.0018 & $d n_{\mathrm{s}} / d \ln k$ & -0.0055 \\
\hline$N_{\mathrm{MI}}$ & 24.3 & $N_{\mathrm{MI}}$ & 27.8 \\
$m_{s} / H_{s}$ & 0.77 & $m_{s} / H_{s}$ & 0.72 \\
\hline
\end{tabular}

(i) The SUGRA corrections play an important role for every $M_{\mathrm{S}}$ in the allowed regions of Fig. 3

(ii) In contrast to standard and shifted FHI, $\left|d n_{\mathrm{s}} / d \ln k\right|$ is considerably enhanced with $-0.005 \gtrsim d n_{\mathrm{s}} / d \ln k \gtrsim-0.01$ holding in a sizable portion of the parameter space for $v_{G} \sim M_{\mathrm{GUT}}$.

(iii) Unlike the cases of standard and shifted FHI, the constraint of Eq. (21) does not restrict the parameters.

(iv) Similarly to the case of shifted FHI, we can find an acceptable solution fixing $n_{\mathrm{s}}=0.958$ and $v_{G}=M_{\mathrm{GUT}}$. Some key inputs and outputs of this solution are arranged in Table 1

\section{Conclusions}

We presented a recently proposed 16 cosmological scenario tied to two bouts of inflation: a GUT scale FHI which reproduces the current data on $P_{\mathcal{R}}$ and $n_{\mathrm{s}}$ within the power-law $\Lambda \mathrm{CDM}$ cosmological model and generates a restricted number of efoldings $N_{\mathrm{HI} *}$ followed by an intermediate scale MI which generates the residual number of e-foldings. We assumed that the inflaton of MI is a string axion which remains naturally almost massless during FHI (and the inter-inflationary period). We considered extra restrictions on the parameters of the model originating from the following:

(i) The resolution of the horizon and flatness problems of the standard hot big bang cosmology.

(ii) The requirements that FHI lasts long enough to generate the observed primordial fluctuations on all the cosmological scales and that these scales are not re-processed by the subsequent MI.

(iii) The limit on the running of the spectral index.

(iv) The naturalness of MI.

(v) The homogeneity of the present universe.

(vi) The complete randomization of the string axion during FHI. 
Fixing the spectral index to its central value, we concluded the following:

(i) In the case of standard FHI, relatively large values of the dimensionless parameter $\kappa$ and the GUT breaking VEV $v_{G}$ are required and $10 \lesssim N_{\mathrm{HI} *} \lesssim 21.7$.

(ii) In the shifted [smooth] FHI case, identification of the GUT breaking VEV with the SUSY GUT scale is possible provided that $N_{\mathrm{HI} *} \simeq 21\left[N_{\mathrm{HI} *} \simeq 18\right]$.

In all three versions of hybrid inflation studied here with $n_{\mathrm{s}}$ near its central value and, in the smooth FHI case, $v_{G} \sim M_{\mathrm{GUT}}$, MI of the slow-roll type with $m_{s} / H_{s} \sim 0.6-0.8$ and a very mild tuning (of order 0.01 ) of the initial value of the string axion produces the additional number of e-foldings required for solving the horizon and flatness problems of standard hot big bang cosmology. Therefore, MI complements successfully FHI.

Note that MI naturally assures a low reheat temperature. As a consequence, baryogenesis is made more difficult. In particular, therma 37 or non-therma 38 leptogenesis won't work since the reheat temperature is very low for the nonperturbative electroweak sphalerons to operate. However, it is not impossible to achieve 39 adequate baryogenesis within a larger scheme with (large) extra dimensions. Let us also mention that, due to the presence of MI, the gravitino constraint 40 on the reheat temperature of FHI and the potential topological defect problem of standard FHI can be significantly relaxed or completely evaded. Our set-up is beneficial for MI too, since, due to its low inflationary scale, this model cannot account for the observed primordial fluctuations (unless a special mechanism 11 is employed).

\section{Acknowledgments}

We thank K. Dimopoulos and R. Trotta for discussions. This work has been supported by the European Union under the contracts MRTN-CT-2004-503369 and HPRN-CT-2006-035863 as well as by the PPARC research grant PP/C504286/1.

\section{References}

1. D.N. Spergel et al., astro-ph/0603449.

2. G. Lazarides, hep-ph/0011130; R. Jeannerot, S. Khalil and G. Lazarides, hep-ph/01 06035.

3. E.J. Copeland, A.R. Liddle, D.H. Lyth, E.D. Stewart and D. Wands, Phys. Rev. D 49, 6410 (1994).

4. G.R. Dvali, Q. Shafi and R.K. Schaefer, Phys. Rev. Lett. 73, 1886 (1994); G. Lazarides, R.K. Schaefer and Q. Shafi, Phys. Rev. D 56, 1324 (1997).

5. V.N. Şenoğuz and Q. Shafi, Phys. Lett. B 567, 79 (2003); ibid. 582, 6 (2003).

6. L. Boubekeur and D. Lyth, J. Cosmol. Astropart. Phys. 07, 010 (2005).

7. M. Bastero-Gil, S.F. King and Q. Shafi, hep-ph/0604198.

8. M. ur Rehman, V.N. Şenoğuz and Q. Shafi, Phys. Rev. D 75, 043522 (2007).

9. B. Garbrecht, C. Pallis and A. Pilaftsis, J. High Energy Phys. 12, 038 (2006).

10. G. Lazarides and A. Vamvasakis, arXiv:0705.3786.

11. R.A. Battye, B. Garbrecht and A. Moss, J. Cosmol. Astropart. Phys. 09, 007 (2006). 
12. H.B. Nielsen and P. Olesen, Nucl. Phys. B61, 45 (1973); T.W.B. Kibble, G. Lazarides and Q. Shafi, Phys. Lett. B 113, 237 (1982).

13. G. Lazarides, Q. Shafi and T.F. Walsh, Nucl. Phys. B195, 157 (1982).

14. J. Rocher and M. Sakellariadou, J. Cosmol. Astropart. Phys. 03, 004 (2005).

15. R. Jeannerot and M. Postma, J. High Energy Phys. 05, 071 (2005).

16. G. Lazarides and C. Pallis, hep-ph/0702260.

17. P. Binétruy and M.K. Gaillard, Phys. Rev. D 34, 3069 (1986); F.C. Adams, J.R. Bond, K. Freese, J.A. Frieman and A.V. Olinto, ibid. 47, 426 (1993); T. Banks, M. Berkooz, S.H. Shenker, G.W. Moore and P.J. Steinhardt, ibid. 52, 3548 (1995); R. Brustein, S.P. De Alwis and E.G. Novak, ibid. 68, 023517 (2003).

18. G. Lazarides, C. Panagiotakopoulos and Q. Shafi, Phys. Rev. Lett. 56, 432 (1986).

19. N. Ganoulis, G. Lazarides and Q. Shafi, Nucl. Phys. B323, 374 (1989).

20. R. Jeannerot, S. Khalil, G. Lazarides and Q. Shafi, J. High Energy Phys. 10, 012 (2000).

21. G. Lazarides and C. Panagiotakopoulos, Phys. Rev. D 52, 559 (1995); G. Lazarides, C. Panagiotakopoulos and N.D. Vlachos, ibid. 54, 1369 (1996); R. Jeannerot, S. Khalil and G. Lazarides, Phys. Lett. B 506, 344 (2001).

22. V.N. Şenoğuz and Q. Shafi, Phys. Rev. D 71, 043514 (2005); hep-ph/0512170.

23. G.'t Hooft, Nucl. Phys. B79, 276 (1974); A. Polyakov, JETP Lett. 20, 194 (1974); J.P. Preskill, Phys. Rev. Lett. 43, 1365 (1979).

24. G. Lazarides, Q. Shafi and W.P. Trower, Phys. Rev. Lett. 49, 1756 (1982).

25. Ya.B. Zeldovich, I.Yu. Kobzarev and L.B. Okun, JETP (Sov. Phys.) 40, 1 (1975).

26. T.W.B. Kibble, J. Phys. A 9, 387 (1976).

27. G. Lazarides, Lect. Notes Phys. 592, 351 (2002); B.A. Bassett, S. Tsujikawa and D. Wands, Rev. Mod. Phys. 78, 537 (2006); G. Lazarides, J. Phys. Conf. Ser. 53, 528 (2006).

28. A. Linde, J. High Energy Phys. 11, 052 (2001).

29. C.P. Burgess, R. Easther, A. Mazumdar, D.F. Mota and T. Multamaki, J. High Energy Phys. 05, 067 (2005).

30. U. Seljak, A. Slosar and P. McDonald, J. Cosmol. Astropart. Phys. 10, 014 (2006).

31. G. Ballesteros, J.A. Casas and J.R. Espinosa, J. Cosmol. Astropart. Phys. 03, 001 (2006).

32. A.A. Starobinsky and J. Yokoyama, Phys. Rev. D 50, 6357 (1994); E.J. Chun, K. Dimopoulos and D. Lyth, ibid. 70, 103510 (2004).

33. M. Dine, L. Randall and S. Thomas, Phys. Rev. Lett. 75, 398 (1995); M.K. Gaillard, H. Murayama and K.A. Olive, Phys. Lett. B 355, 71 (1995).

34. G. Lazarides, R. Ruiz de Austri and R. Trotta, Phys. Rev. D 70, 123527 (2005); G. Lazarides, Nucl. Phys. B, Proc. Suppl. 148, 84 (2005).

35. J.C. Pati and A. Salam, Phys. Rev. D 10, 275 (1974).

36. G. Lazarides, M. Magg and Q. Shafi, Phys. Lett. B 97, 87 (1980).

37. M. Fukugita and T. Yanagida, Phys. Lett. B 174, 45 (1986).

38. G. Lazarides and Q. Shafi, Phys. Lett. B 258, 305 (1991); G. Lazarides, C. Panagiotakopoulos and Q. Shafi, ibid. 315, 325 (1993), (E) 317, 661 (1993); G. Lazarides, Q. Shafi and N.D. Vlachos, ibid. 427, 53 (1998); G. Lazarides and N.D. Vlachos, ibid. 459, 482 (1999).

39. K. Benakli and S. Davidson, Phys. Rev. D 60, 025004 (1999).

40. M.Yu. Khlopov and A.D. Linde, Phys. Lett. B 138, 265 (1984); J. Ellis, J.E. Kim and D.V. Nanopoulos, ibid. 145, 181 (1984).

41. K. Dimopoulos and G. Lazarides, Phys. Rev. D 73, 023525 (2006); G. Lazarides, AIP Conf. Proc. 881, 144 (2007). 